\title{
Editorial
}

Nephrology

\section{Racial Disparities in End-of-Life Issues in Patients with Chronic Kidney Disease}

\author{
Keith C. Norris Susanne B. Nicholas \\ Department of Medicine, David Geffen School of Medicine at UCLA, Los Angeles, Calif., USA
}

Chronic kidney disease (CKD) affects nearly $15 \%$ of adults in the United States (US) and is associated with a progressive increase in the risk of premature morbidity and mortality as kidney function deteriorates [1]. Patients receiving replacement therapy (RRT) are at the highest risk for mortality, and end-of-life (EOL) issues including advanced care planning (ACP) and hospice care have been increasingly promoted in this subset of patients [2]. In fact, during the time period 2000-2012, the percentage of Medicare beneficiaries with end-stage renal disease (ESRD) admitted to the intensive or coronary care unit during the last 90 days of life rose from 50 to $63 \%$, while those who died in the hospital decreased from 47 to $41 \%$ [2]. Additionally, the percentage of patients who discontinued maintenance dialysis treatments before death increased from 19 to $25 \%$, while patients with ESRD receiving hospice care at the time of death increased from 11 to $25 \%$, suggesting a substantial increase in the use of ACP and EOL options.

Of note, for the subset of patients who opted to discontinue dialysis treatments, the use of hospice services between 2004 and 2012 increased substantially (from 59 to $80 \%$ ), leading to a significant reduction in Medicare costs per person during the last week of life [2]. While major gains that have been made in the use of hospice services

\section{KARGER}

(c) 2016 S. Karger AG, Basel

0250-8095/16/0441-0081\$39.50/0

E-Mail karger@karger.com

www.karger.com/ajn have been seen across all racial/ethnic groups, Black and Hispanic patients still remain less likely to discontinue dialysis or utilize hospice care compared to their white peers [2].

The discussion of EOL is always challenging, but patients receiving RRT have already transitioned through many of the hurdles that accompany the period of preESRD care. By contrast, patients with advanced stages of $\mathrm{CKD}$ who are not receiving RRT present a unique dilem$\mathrm{ma}$, as their risk for a major adverse event is also great, but many are overwhelmed by concerns regarding progression to RRT, deteriorating health, struggling with dietary and medication recommendations, coordinating care for associated comorbidities related to their underlying disease(s), and selecting an RRT modality [3]. In addition, they are often trying to navigate these multiple complex issues in the face of declining cognitive functioning from the underlying disease, progressive azotemia, depression, and/or polypharmacy [3]. Thus, much less is known about how to best approach EOL care issues in patients with CKD who are not yet receiving RRT. To further complicate the issue, there are many patient-level factors such as language, race/ethnicity, religion, age and socioeconomic status that may further influence receptivity to and individual prioritization of EOL issues. 
A recent survey of 431 physicians noted $80 \%$ were likely, or extremely likely to discuss conservative care with patients with stages $4-5$ CKD, but less than $50 \%$ were likely, or extremely likely to discuss palliative care [4]. In this issue of the American Journal of Nephrology, Eneanya et al. [5] explore EOL care among patients with advanced $\mathrm{CKD}$ prior to decisions regarding the initiation of dialysis with a focus on potential racial differences in EOL communication, ACP, and care preferences. In a cross-sectional analysis of over 150 patients with stages 4-5 CKD, they found that Black patients were less likely to communicate EOL preferences and had less knowledge of hospice than their white counterparts, even after adjusting for factors such as age, education and income. Interestingly, they found no racial differences in distrust of providers, or in spiritual/religious or cultural beliefs. The reasons for the racial differences reported in this study are not clear, but the observation has important implications in caring for a diverse population of patients with CKD.

Effectively engaging patients with CKD in EOL discussions requires a delicate balance of multiple patient level factors. For instance, age may play an important role in EOL decisions as younger persons may feel a greater sense of immortality and may be more reticent to engage in such discussions. However, possibly more powerful than issues such as age, income, education, race/ethnicity or religion may be issues related to classism and wealth, and potential residual confounders that may not be captured by traditional measures of socioeconomic status. Impoverished individuals are more likely to be immersed with issues related to day-to-day survival [6]. By contrast, wealthy individuals are likely to spend more of their time on estate planning and on plans to ensure transition of their wealth to their loved ones [6]. Thus, they are much more likely to be familiar and comfortable with EOL issues, and one might hypothesize that they would be more open to discussions around EOL options. In older persons, increased wealth is associated with lower symptom burden, likely mediated through increased access to health care and other social services [7] and this may further free the cognitive space required to prioritize EOL discussions. A recent report found white households in Los Angeles had a median net worth (assets minus debt) of $\$ 355,000,100$ times higher than households of Mexicans and US Blacks at only $\$ 3,500$ and $\$ 4,000$, respectively [8]. Such a huge gap could have a tremendous influence on the racial/ethnic divide of how families prioritize estate and EOL concerns.

Despite not having data on wealth, the findings presented by Eneanya et al. [5] provide important insights into patient preferences at advanced stages of CKD, prior to RRT. Given advanced CKD is more prevalent among persons with low socioeconomic status and/or racial/ethnic minorities, the nephrology community needs to give extra thought to how to best approach the discussion of EOL care in these diverse and overly burdened cohorts. Since the topic of EOL care may be difficult to introduce closer to the need for RRT as patients are inundated with the multiple competing demands associated with CKD progression, concerted efforts should be made to start such discussions at earlier stages of CKD, such as stage 3 and for using a team approach $[9,10]$.

In summary, the findings presented by Eneanya et al. [5] should serve as a reminder to CKD providers and healthcare teams to be sensitive to the potential for culture, class, generation and other elements to influence patient views and differing nuances of apprehensions about EOL discussions. This sensitivity could allow for more effective and compassionate dialog and enhance the engagement of greater numbers of patients with advancing $\mathrm{CKD}$ in EOL and ACP.

\section{Disclosure Statement}

K.C.N. is supported by NIH grants UL1TR000124, P30AG021684 and P20-MD000182. S.B.N. is supported by NIH grants 1DP3 DK094311 and UL1TR000124. The content is solely the responsibility of the authors and the authors declare that they have no relevant financial interests and no conflict of interest.

\section{References}

1 Coresh J, Astor BC, Greene T, Eknoyan G, Levey AS: Prevalence of chronic kidney disease and decreased kidney function in the adult US population: third national health and nutrition examination survey. Am J Kidney Dis 2003;41:1-12.

2 USRDS: US Renal Data System: 2015 USRDS Annual Data Report: Epidemiology of Kidney
Disease in the United States. Bethesda, National Institutes of Health, National Institute of Diabetes and Digestive and Kidney Diseases, 2015.

3 Kahn LS, Vest BM, Madurai N, Singh R, York TR, Cipparone CW, Reilly S, Malik KS, Fox $\mathrm{CH}$ : Chronic kidney disease (CKD) treatment burden among low-income pri- mary care patients. Chronic Illn 2015;11: 171-183.

4 Parvez S, Abdel-Kader K, Pankratz VS, Song MK, Unruh M: Provider knowledge, attitudes, and practices surrounding conservative management for patients with advanced CKD. Clin J Am Soc Nephrol 2016;11:812820 
5 Eneanya ND, Wenger JB, Waite K, Crittenden S, Hazar DB, Volandes A, Temel JS, Thadhani R, Paasche-Orlow MK: Racial disparities in end-of-life communication and preferences among chronic kidney disease patients. Am J Nephrol 2016, in press.

6 Payne RK, Blair T: A Framework for Understanding Poverty. Highlands, Aha! Process, 2005.
7 Silveira MJ, Kabeto MU, Langa KM: Net worth predicts symptom burden at the end of life. J Palliat Med 2005;8:827-837.

8 De La Cruz-Viesca M, Chen Z, Ong PM, Hamilton D, William Jr AD: The Color of Wealth in Los Angeles. Los Angeles, Duke University, The New School, and The University of California, 2016.
9 Davison SN, Levin A, Moss AH, et al: Executive summary of the KDIGO controversies conference on supportive care in chronic kidney disease: developing a roadmap to improving quality care. Kidney Int 2015;88:447-459. 10 O'Hare AM, Szarka J, McFarland LV, et al: Provider perspectives on advance care planning for patients with kidney disease: whose job is it anyway? Clin J Am Soc Nephrol 2016; 11:855-866. 